\title{
La comunicación entre los ciudadanos y los representantes políticos a través del correo electrónico. Análisis del caso de la delegación española en el Parlamento Europeo durante los años 2013 y 2014
}

Communication between citizens and political representatives by email. A case analysis of the Spanish delegation in the European

\section{Parliament for 2013 and 2014}

Francisco Javier Puchades Arce. Universidad CEU Cardenal Herrera (javierpuchades@hotmail.com)

Recibido el: 18/10/15 - Aceptado el: 18/03/16

Resumen:

La participación ciudadana en los asuntos públicos ha encontrado un nuevo campo de expansión en las tecnologías de la información, favoreciendo la implicación política de las personas. El presente trabajo analiza la comunicación entre ciudadanos y eurodiputados españoles a través del correo electrónico. Para ello, el investigador ha dirigido un grupo de individuos que, durante tres oleadas, se han comunicado con los europarlamentarios a través de esta vía. Junto con el análisis cuantitativo se ha realizado un análisis de contenido para evaluar la calidad de las respuestas. Las conclusiones señalan bajos porcentajes de respuesta en el uso que los eurodiputados hacen de esta herramienta. No obstante, aquellos que responden muestran un elevado interés en resolver la cuestión planteada por su interlocutor.

Palabras clave:

Interacción; Email; Ciudadanos; Diputados; Parlamento Europeo.

Abstract:

Participation in public affairs has been expanded by new information technologies. Citizens have found more opportunities to interact with their public representatives. It might lead to an enhancement of people's political involvement. This paper analyzes the relationship between citizens and Spanish MEPs via e-mail. The researcher has conducted a group of people who, along three temporary waves, have adressed emails to the Spanish MEPs. Apart from observing the percentage of answers, a content analysis based on categories has been applyed in order to assess the quality of the answers. The findings point to low response rates when using email to communicate with citizens. However, those MEPS answering put a great deal of effort in resolving the questions asked by citizens.

Keywords:

Interaction; Email; Internet; Citizens; MEPs; European Parliament. 


\section{Introducción}

El establecimiento y posterior fortalecimiento de los sistemas democráticos se ha revelado como una de las claves de la prosperidad de las sociedades occidentales tras la finalización de las dos Guerras Mundiales del siglo XX. Sus sólidas estructuras han permitido a la población alcanzar niveles de organización, bienestar, convivencia y conocimiento que se habían visto interrumpidos o mermados en etapas históricas anteriores. Sin embargo, tras sólidas bases de madurez y ejercicio alcanzadas tanto por los ciudadanos como por sus gobernantes, la democracia ha empezado a mostrar síntomas de insuficiencia y de estar atravesando por un proceso de crisis, si bien algunos de estos síntomas se habían hecho evidentes desde las primeras etapas del sistema.

La posición de monopolio adquirida por los partidos políticos dentro del sistema de la democracia representativa ha sido interpretada como un inconveniente por parte de autores como De Vega García (1996: 94), Sanmartí (2003: 92) o Maravall (2003: 30). El Parlamento, que debería servir como escenario de deliberación y debate desde donde se ejerce la potestad legislativa del Estado y la función de control al Gobierno, ha sido desposeído de esa función frente a la maquinaria de los grandes partidos políticos. La actividad parlamentaria se ha trasladado a los despachos y comités de dirección de las formaciones que, marcadas por una férrea disciplina de voto interna, han limitado la discusión y debate en el escenario parlamentario (Sinova y Tusell, 1997: 82; Pindado, 1999: 31).

En este proceso de crisis de la democracia representativa también ha jugado un papel fundamental la televisión, como gran medio de comunicación de masas del siglo XX. El empoderamiento de la cultura de la imagen (Postman, 2001:12) y el deseo de los Gobiernos y los partidos políticos por acceder a grandes audiencias ha generado la aparición de una democracia mediática, dominada por la simplificación y estandarización del discurso político (Hart, 1994: 118; Sartori, 1998:55; Neveu, 1998: 154; Rospir, 1999: 75; Gingras, 1998: 35) y por el gobierno de los sondeos (Muñoz Alonso, 1999: 45; Sartori, 1998: 78; Gómez Fernández, 1999: 199).

Fallows (1996: 31) considera que los medios de comunicación, en especial la televisión, son los grandes responsables de la pérdida de confianza de los ciudadanos en los partidos políticos y en las instituciones públicas. Norris (2003: 13) elude centrar la responsabilidad en los medios, pero no duda que ese proceso de alejamiento y erosión de la confianza ciudadana se ha producido a causa de la mala actuación de los Gobiernos (Newton y Norris, 2000). Es lo que Putnam (2003: 639) identifica como pérdida del capital social, y que atribuye al papel de espectadores que han adquirido los ciudadanos dentro de la maquinaria del sistema democrático.

Este declive de la confianza en las instituciones públicas y en los partidos políticos va acompañado del florecimiento de nuevas formas de capital social. Pérez Díaz (2003: 468) se refiere a ellas como "asociacionismo del tercer sector", es decir causas alejadas de valores estrictamente políticos y económicos, y mucho más relacionadas con la realización 
personal o los nuevos estilos de vida. En este sentido, Torcal (1992: 118) y Morales (2005: 76) apuntan a un desplazamiento del conflicto social desde los valores materialistas hasta los posmaterialistas. En este proceso, una vez consolidadas ciertas conquistas en el terreno social y económico, han ido ganando peso otras causas como la libertad de expresión, el ecologismo, la igualdad entre hombres y mujeres, o la lucha por los derechos de los más desfavorecidos. Putnam (1995:70), en otra de sus obras, ya anuncia que el deterioro del compromiso cívico va acompañado del florecimiento de nuevas organizaciones desmovilizadas políticamente pero comprometidas con otras causas como el medio ambiente o el feminismo.

Desde su aparición, las tecnologías de la información, y las herramientas de comunicación en internet de forma particular, se han convertido en un elemento esencial para el funcionamiento de estas organizaciones terciarias. Los lazos que unen a sus miembros son más débiles y distantes, si bien la cada vez mayor penetración de internet ha contribuido a generar un compromiso cívico privado solitario, aunque no por ello menos efectivo (Papacharisi, 2009: 32).

Prácticamente desde los primeros pasos de internet como elemento de comunicación social, los movimientos sociales han visto en las tecnologías de la información un elemento determinante tanto para la movilización como para su organización interna. Castells (2003: 106) describe la forma en la que Movimiento Zapatista consiguió superar el bloqueo informativo al que fue sometido por parte del Ejecutivo Mexicano en la década de los 90 gracias al uso de listas de distribución y redes de comunicación en internet.

El propio Castells (2003: 184) documenta otros ejemplos, si bien sitúa el movimiento antiglobalización como el paradigma capaz de explotar internet como elemento de coordinación y comunicación dentro de una organización colectiva. Las movilizaciones de Seattle en 1999, coincidiendo con la clausura del encuentro de la Organización Mundial del Comercio, se han sucedido posteriormente donde, aunque en escenarios diferentes, el papel de internet ha resultado igualmente crucial. La red ha permitido generar unas lógicas de funcionamiento basadas en la horizontalidad, la ausencia de jerarquía, el acceso abierto, la participación directa y la toma de decisiones por consensos entre los activistas. Es lo que Juris (2006: 434) define como un "laboratorio de democracia" capaz de dar paso a "una nueva forma de hacer política".

Una década después que el movimiento antiglobalización explotase, con éxito para sus integrantes, la capacidad organizativa y de movilización que les ofrecían las herramientas de comunicación de internet, centenares de grupos y activistas volvieron a recurrir a las tecnologías de la información durante la llamada Primavera Árabe. Martínez (2011:3) e Higueras (2011) documentan el uso de las redes sociales Facebook y Twitter en las actividades de comunicación, organización y movilización de los protagonistas de aquellas revueltas, mientras que otros autores como Morozov (2011: 57) exhiben una postura mucho más crítica al recordar como Twitter y Facebook fueron empleados como vía para silenciar, oprimir y perseguir a los activistas del Movimiento Verde en Irán en 2009. 
En la misma línea, Arroyo (2012) y Gutiérrez-Rubí (2008) advierten de que las empresas que gestionan las redes sociales son entidades privadas que pueden hacer lo que deseen dentro de sus dominios, desde suprimir cuentas hasta eliminar rastros históricos de archivos. Asimismo, alertan del nacimiento de una especie de activismo de sofá que mide la fuerza de las causas en función del número de apoyos en las redes sociales.

El universo de internet también está muy presente en la génesis del movimiento 15M en España. De hecho, Scolari (2009) sitúa su origen en la corriente generada en la red tras conocer las intenciones del Gobierno con la llamada 'Ley Sinde’. Circularon manifiestos y se crearon etiquetas en Twitter que desembocaron en una manifestación celebrada el viernes 4 de diciembre de 2009 en Madrid, a la que también se sumaron colectivos desencantados con la gestión y los efectos de la crisis económica sobre la población (Ferreras, 2011: 3). Sobre este punto, Dans (2011) recuerda que la red bullía con miles de tuits, grupos de Facebook y entradas en blogs que, meses después, se trasladaron al escenario offline cuando Democracia Real Ya convocó manifestaciones para la tarde del 15 de mayo de 2011 en varias ciudades españolas. Ferreras (2011: 3) y Congosto (2011) insisten en la importancia de la cobertura que dieron los medios tradicionales, especialmente la televisión, en la amplificación del impacto de las movilizaciones del 15M, mientras que Sampedro y Sánchez Duarte (2011) apuntan al protagonismo esencial de los espacios colaborativos 2.0 que las redes sociales aportaron al movimiento.

Además de la capacidad de movilización y coordinación que han demostrado las redes sociales, son varios los autores que consideran que aquello que se acrecienta con el 15M es la pérdida del monopolio de los partidos políticos, algo que ya se había empezado a producir con la aparición de los nuevos movimientos sociales (Gutiérrez Rubí, 2011 y Orihuela, 2011). Es, tal como señala Reig (2011), un ejercicio de empoderamiento de la ciudadanía.

Esta corriente entronca con el pensamiento de aquellos autores que, prácticamente desde la aparición de internet, le han otorgado un protagonismo especial como elemento para revitalizar la democracia a través de la mayor participación política de los ciudadanos en los asuntos públicos y de la creación de canales de comunicación más estrechos entre los individuos y sus representantes públicos (Negroponte, 1995: 271; Rheingold, 1993: 60; Grossman, 1995: 165; Norris, 2003: 34). No obstante, la investigación realizada por Dader (2003: 312) y Campos (2011:385) en el marco del Congreso de los Diputados español revela bajos porcentajes de respuesta en la comunicación entre los ciudadanos y los diputados a través del email. Asimismo, los estudios realizados sobre el medio Twitter revelan niveles de interacción muy bajos en forma de conversación (intercambio pregunta-respuesta) entre los candidatos y sus seguidores en las elecciones generales españoles de 2011 (Zurutuza, 2012), las elecciones al Parlamento Vasco de 2012 (Cebrián, Vázquez y Olabarrieta, 2013: 60) y los comicios al Parlamento Andaluz del mismo año (Rodríguez Vives, Congosto y Giura, 2012: 40).

En las elecciones al Parlamento Europeo de 2014, la consultora Porter Novelli analizó la interacción de los cabeza de lista de los partidos con sus seguidores a través de Twitter. Los resultados indicaban que solo los número 1 de l'Esquerra pel Dret a Decidir y de Los Pueblos Deciden dedicaban más del 10 por ciento de sus mensajes a responder a otros tuiteros (13,3 por 
ciento y 10,5 por ciento, respectivamente). El resto se quedaba muy por debajo de esas cifras. El estudio apuntaba que los candidatos empleaban la red mayoritariamente para difundir sus ideas o sus actos de campaña, pero no para conversar con sus seguidores ${ }^{1}$.

En la red social Facebook la tendencia es similar, tanto en los resultados del trabajo realizado por Túñez y Sixto (2011: 19-20) en el marco del Congreso de los Diputados de España como en la investigación de Dapena y Rúas Araújo (2011: 94) centrada en el caso de los diputados del Parlamento Gallego. Los estudios de Lilleker y Jackson (2009: 12) en el marco del Parlamento Británico y de Small (2010: 42) en entorno del Parlamento Federal Canadiense muestran una tendencia muy parecida.

A pesar del potencial de la web 2.0 para interactuar, producir e intercambiar contenido (O'Reilly, 2005; Lilleker y Jackson, 2008) la literatura existente revela que los representantes políticos no están empleando las herramientas de comunicación de internet con actitud 2.0, es decir con la vocación de "potenciar su capacidad de abrirse, de hablar con la ciudadanía y de escucharla, de compartir sus opiniones” (Fagés Ramió, 2008: 22). La tecnología ha creado canales para la participación ciudadana en los asuntos públicos que, no obstante, se siguen utilizando de forma mayoritariamente unidireccional en el apartado que se refiere a la comunicación entre los ciudadanos y sus representantes políticos, limitando así la capacidad de empoderamiento ciudadano a la que se hacía referencia anteriormente.

\section{Metodología}

La investigación realizada tiene como objetivo analizar la relación entre los ciudadanos y los representantes políticos a través del correo electrónico. Para ello, se ha tomado como punto de referencia el marco teórico descrito en el apartado anterior en el que, como se ha apuntado, ya existen trabajos capaces de aportar conclusiones relevantes en esta parcela del conocimiento. Sobre estas bases, la novedad que aporta la presente investigación reside en la combinación de metodología cuantitativa y cualitativa para analizar esta relación desde una doble vertiente. Los trabajos previos se detienen en el análisis numérico de la interacción entre los representantes políticos y los ciudadanos, mientras que el presente trabajo aporta una actualización metodológica a través del análisis de contenido sobre la base de los mensajes que conforman esa interacción.

Asimismo, la cercanía temporal del período en el cual se ha ejecutado el trabajo de campo (años 2013 y 2014) respecto al momento presente permite ofrecer una fotografía actualizada sobre el objeto de la cuestión.

El diseño metodológico ha partido del planteamiento de una serie de cuestiones de la investigación, que enlazan con lo que Fagés Ramió (2008: 22) define como actitud 2.0: ¿Cuál es el grado de respuesta de los representantes políticos hacia

Fuente http://www.valenciaplaza.com/ver/130964/los-candidatos-a-las-europeas-solo-usan-twitter-para-hacer-propaganda.html (Consultado el: 14/05/2014). 
los ciudadanos a través del email? ¿Qué clase de respuesta ofrecen los representantes públicos? ¿En qué medida estos mismos representantes se esfuerzan no solo en responder, sino también en resolver la cuestión planteada por la ciudadanía y llevar esa interacción más allá del par pregunta-respuesta?

Para responder a estas cuestiones, la investigación se ha centrado en el escenario de la delegación española en el Parlamento Europeo. La justificación de esta elección reside en que la estructura, representantes y funciones del Europarlamento encajan con el planteamiento del trabajo. Si el objetivo que se propone es averiguar si los representantes políticos están utilizando las tecnologías de la información para relacionarse con el ciudadano se debe colocar el foco investigador sobre un conjunto de representantes políticos (los eurodiputados españoles, en este caso), analizarlo y ver qué sucede cuando los ciudadanos se dirigen a ellos a través de estas herramientas.

La justificación del uso del email como herramienta para analizar esta relación reside, en primer lugar, en su amplia penetración social. El email sigue siendo, tras las aplicaciones de mensajería instantánea, el servicio más utilizado por los internautas. El 74,2 por ciento de los usuarios de internet asegura haberlo utilizado en el último mes (Asociación para la Investigación de los Medios de Comunicación. AIMC, 2015).

En segundo lugar, el email permite mantener una relación interactiva plena en la que los papeles de emisor y receptor son altamente intercambiables, el control sobre el contenido del mensaje reside en ambos y la dirección de la comunicación circula en ambas direcciones (Vela Delfa, 2005: 730, Yus Ramos, 2010: 728).

Una vez definido el marco de estudio, los objetivos y el escenario a analizar, se han planteado dos hipótesis de investigación.

1.- Los diputados de la delegación española en el Parlamento Europeo no emplean el correo electrónico para mantener una relación directa e interactiva con los ciudadanos que se dirigen a ellos a través de esta vía.

El planteamiento de esta hipótesis se justifica en que toda la literatura y trabajos que se han consultado, tal como se ha descrito en el apartado número uno, apuntan en esta línea.

2.- Los diputados de los grupos parlamentarios minoritarios de la delegación española en el Parlamento Europeo realizan un uso más intenso del email respecto a los grupos mayoritarios para relacionarse e interactuar con la ciudadanía que se dirige a ellos a través de esta vía. Sobre este punto, se han considerado grupos mayoritarios el Grupo del Partido Popular Europeo y el Grupo de la Alianza Progresista de Socialistas y Demócratas en el Parlamento Europeo, al ser ambos los que cuentan con un mayor número de representantes dentro de la delegación española. Al resto de grupos se le ha dado la consideración de minoritarios.

Para establecer la segunda hipótesis se han seguido las teorías de Lilleker y Jackson (2008 y 2009: 20) y Norris (2003: 34) según las cuales los partidos minoritarios, con unos recursos económicos y una dimensión mediática mucho más limitada, 
explotan en mayor medida las herramientas de comunicación de internet como vía para sortear la menor atención que reciben por parte de los medios convencionales. Asimismo, se han seguido la tesis de Gibson y Ward (1999: 361), según las cuales internet ayuda a reducir el problema de la distancia entre eurodiputados y electores. Internet permite dar visibilidad a su trabajo y a su figura, particularmente en un escenario en el que los medios tradicionales generan poca cobertura sobre la actividad que desarrollan en el Parlamento Europeo.

Para cumplir con los objetivos planteados se ha diseñado una triangulación metodológica basada en la combinación de técnicas cuantitativas y cualitativas para analizar tanto los índices de respuesta de los integrantes de la muestra como el contenido de esas respuestas.

En primer lugar, se ha diseñado un experimento a través del envío de correos electrónicos a todos los eurodiputados españoles integrados en la muestra construida para desarrollar la investigación. El objetivo ha sido situar a los representantes políticos ante una situación real, es decir ante ciudadanos que se dirigían a ellos a través del email para interpelarles y trasladarles una serie de cuestiones.

El primer paso dentro de la metodología del análisis cuantitativo ha sido definir el universo de la investigación, formado por los 54 diputados que componen la delegación española en el Parlamento Europeo. Dentro del universo, la muestra está formada por aquellos diputados que hacen pública una cuenta de email a través de la cual dirigirse a ellos. En este sentido, conviene puntualizar que la muestra ha venido condicionada por la decisión de los parlamentarios de facilitar una cuenta de email a través de las 3 fuentes oficiales que se han consultado para construir la muestra, y que son las siguientes:

1.- Fuente A: La ficha personal de cada diputado alojada en el sitio web del Europarlamento.

2.- Fuente B: Si los diputados no hacían pública una cuenta de email a través de la fuente A se ha procedido a buscar su dirección de correo electrónico en el sitio web de su partido político o grupo parlamentario tanto a nivel nacional como autonómico.

3.- Fuente C: Si tampoco se obtiene el contacto a través de esta fuente se ha procedido a buscar la dirección electrónica en el sitio web del partido a nivel provincial o local.

Se trata, en todos los casos, de fuentes públicas, oficiales, accesibles a todos y en las que se identifica al integrante de la muestra en su condición de diputado. El empleo de tres fuentes aporta a la metodología un criterio de exhaustividad cuyo objetivo ha sido el de poder contar con una muestra lo más amplia posible. Así, se ha intentando localizar las cuentas de email de los eurodiputados españoles en aquellas fuentes oficiales, públicas y accesibles a todos los ciudadanos. 
El trabajo de campo se ha planteado en 3 oleadas separadas en el tiempo a lo largo de los años 2013 y 2014. Antes y durante cada una de las oleadas se han consultado de nuevo las fuentes para actualizar la muestra y registrar los posibles cambios acontecidos por las altas o bajas de eurodiputados.

La primera oleada se ha ejecutado entre los meses de marzo y junio de 2013. La segunda entre noviembre y diciembre de 2013, y enero de 2014. La tercera y última oleada se ha desarrollado entre los meses de septiembre y diciembre de 2014. La justificación de la elección de tres oleadas temporales reside en la capacidad de aportar una perspectiva más amplia de la realidad objeto de estudio. Asimismo, trabajar con una línea temporal amplia ha permitido analizar la evolución registrada en el número de respuestas y establecer una comparación entre ellas a partir de las variables estudiadas.

Para realizar los envíos se ha conformado un grupo de 30 ciudadanos reales, que han sido dirigidos por el investigador y han aceptado participar en la investigación. Cada uno de ellos ha creado una cuenta de email con su nombre y apellidos a través del proveedor de servicios Gmail, por medio de la cual se han efectuado los envíos a los integrantes de la muestra.

El contenido de los emails dirigidos a los integrantes de la muestra se ha trabajado para personalizarlo al máximo y adaptarlo a su destinatario. Todos los correos electrónicos han sido redactados en un estilo formal y presentan una estructura compuesta por tres partes: encabezamiento/introducción, cuerpo y desenlace/cierre. El objetivo en su elaboración ha sido hacer explícito que se trataba de un mensaje personal, intransferible y dirigido en exclusiva a ese diputado en concreto. En otras palabras, dejar claro a través de ese contenido que no se trataba de un correo más dentro de una cadena de envíos masivos ni de un elemento de spam.

Para conseguir esa personalización se ha trabajado el contenido de los emails en cuatro elementos considerados fundamentales. En primer lugar, a través del asunto, donde se ha realizado un resumen concreto del contenido del mensaje. La personalización se ha buscado, en segundo lugar, en el encabezamiento del email, con el nombre y apellidos del diputado al cual iba dirigido el correo electrónico. Asimismo se ha adaptado el contenido de cada email a los asuntos o materias tratadas por la comisión o comisiones a la que pertenece el diputado. Es decir, el contenido interpelaba al diputado acerca de cuestiones relacionadas exclusivamente con su área de competencia en la cámara. Por último, en el cierre el ciudadano se despide con una fórmula de agradecimiento, su nombre y apellidos, y su DNI. De esta forma, aunque emisor y receptor no se conocen, el ciudadano ofrece a su interlocutor una marca de confianza y seriedad que permite identificarlo y dotarlo de personalidad real durante esa interacción.

Conviene, en este punto, plantear una de las limitaciones de la investigación. Es posible que en algunos casos, y a pesar de los esfuerzos metodológicos para adaptar los mensajes a las competencias de cada uno de los diputados (basándonos en la especialización de las materias que genera la propia cámara a partir de la distribución del trabajo en comisiones par- 
lamentarias), el contenido de los mismos mensajes enviados haya podido influir en el índice de respuesta de los representantes. Es decir, que la falta de conocimiento específico sobre la materia planteada haya motivado la ausencia de respuesta.

Sobre la base de las tres oleadas de envíos se han diseñado dos clases de mensajes diferentes para analizar la respuesta de los diputados ante cada uno de ellos. Para la primera y segunda oleada se han elaborado lo que se ha definido como Mensajes-Desarrollo (MD). Poseen una estructura dividida en tres partes (introducción, cuerpo y desenlace) y en el contenido, tal como se ha explicado anteriormente, se interpela a los diputados acerca de cuestiones concretas relacionadas con el área de competencia dentro de la cámara. Son correos extensos (entre 12 y 18 líneas) que requieren un mínimo de tiempo y dedicación para ser leídos, comprendidos y respondidos por parte del diputado. El emisor, en este caso el ciudadano, demuestra un adecuado nivel de conocimiento del asunto y es posible que el receptor también necesite consultar algún documento o refrescar su memoria para responder. El siguiente ejemplo corresponde a un email enviado por un ciudadano a un integrante de la Comisión de Industria, Investigación y Energía.

Asunto: Estado del reglamento para hacer posible la firma electrónica transfronteriza.

Estimado diputado... (nombre y apellidos del eurodiputado)

Aprovecho su dirección de correo está disponible en la página web del Parlamento Europeo para interpelarle acerca de un asunto de su competencia como representante de la comisión de industria, investigación y energía del Parlamento Europeo. Como sabrá, en España existe una legislación que habilita la firma electrónica para establecer diversos trámites con la administración pública, si bien es cierto que se trata de un procedimiento que pocos ciudadanos utilizan a título individual (...).

Entiendo que es un proceso complejo, sobre todo el reconocimiento de cada uno de los sistemas de firma electrónica de los países miembro pero ¿en qué estado se encuentra este reglamento para hacer posible la firma electrónica transfronteriza? ¿ ¿Es posible que se apruebe en 2014, coincidiendo con el nuevo período de sesiones del PE tras las elecciones mayo? Una reflexión final para los que nos interesa este tema: piensen que, en teoría, tenemos un mercado único y una moneda común, pero en cuanto a los sistema de firma electrónica la UE sigue operando como un conjunto de países individuales.

Un cordial saludo

(Nombre, apellidos y DNI del ciudadano)

En la tercera oleada, el contenido de los Mensajes-Desarrollo se ha sustituido por lo que se ha acordado en definir como Mensajes-Función (MF). Estos mantienen la misma estructura, pero el contenido del email se convierte en mucho más escueto: entre 3 y 6 líneas. Se interpela al diputado acerca de cuestiones relacionadas con su área de competencia dentro de 
la cámara pero se trata, en todos los casos, de preguntas acerca del funcionamiento ordinario de la Eurocámara. Por ejemplo, cómo se puede seguir un pleno a través de internet, dónde es posible descargar el diario de sesiones de una comisión determinada o qué hay que hacer para acudir a presenciar en directo una sesión de una comisión parlamentaria. A diferencia de los MD, los MF requieren un esfuerzo mucho menor por parte del diputado para su lectura y posterior respuesta. Se leen y contestan de forma muy rápida y sencilla. La personalización se mantiene en los mismos términos a través del encabezamiento y del contenido, tal como puede observarse en el siguiente ejemplo del email remitido a un diputado miembro de la Comisión de Desarrollo.

Asunto: Acceder al orden del día de la próxima comisión de desarrollo.

Diputado (nombre y apellidos del diputado), buenos días. Le escribo como integrante de la comisión de desarrollo del Parlamento Europeo. Me gustaría saber de qué manera puedo consultar el orden del día de la próxima comisión de desarrollo del Europarlamento y los temas que se van a debatir en ella. ¿Está disponible en algún documento online o se puede descargar? Aunque la legislatura está recién empezada, entiendo que las comisiones ya están funcionando de forma habitual.

Gracias por su atención

(Nombre, apellidos y DNI del ciudadano)

Los mensajes se han redactado de forma específica para cada una de las oleadas. Todos los mensajes, tanto los MD como los MF, contienen una pregunta clara y concreta que busca la respuesta del diputado. El ciudadano le plantea una cuestión, reforzada con el uso de los signos de interrogación, y espera de él una contestación. Este matiz es importante, ya que uno de los criterios clave para el análisis de contenido categorial que se verá a continuación es identificar si el diputado responde a la cuestión planteada por su interlocutor y la resuelve.

Para el análisis cuantitativo de los índices de respuesta se han empleado operaciones de regla de tres, suma y comparación de porcentajes (cuántos diputados responden a los emails enviados por los ciudadanos en cada una de las oleadas).

En el caso del análisis cualitativo se ha planteado un análisis de contenido categorial. El cuadro 1 contiene las categorías e indicadores que se han empleado para este análisis. Estas categorías e indicadores se han aplicado sobre cada una de las unidades de análisis o muestreo, correspondientes al contenido de los emails de respuesta de los diputados. Las unidades de registro corresponden a las partes mínimas de las unidades de muestreo que se pueden analizar de forma aislada, es decir las frases o palabras clave que ayudan al investigador a determinar la adscripción a cada una de las categorías (Krippendorff, 1990: 82; Bardin, 1986: 91). 
Cuadro 1. Categorías e indicadores para el análisis de contenido categorial

\begin{tabular}{|c|c|c|}
\hline Categoría & Indicador & Descripción \\
\hline \multirow{2}{*}{ RESPUESTA } & Respuesta & Contesta al envío \\
\hline & No respuesta & No contesta al envío \\
\hline \multirow{2}{*}{ ACCIÓN } & Respuesta delegada & Responde un asistente del equipo o grupo del diputado \\
\hline & Respuesta personalizada & El diputado responde personalmente \\
\hline \multirow{3}{*}{ CONTENIDO } & Respuesta sucinta & $\begin{array}{l}\text { El diputado responde, pero no resuelve la cuestión que le formula } \\
\text { su interlocutor }\end{array}$ \\
\hline & Respuesta disociada & $\begin{array}{l}\text { El diputado no resuelve la cuestión planteada. Su respuesta no } \\
\text { tiene nada que ver con el tema planteado por su interlocutor }\end{array}$ \\
\hline & Respuesta argumentada & $\begin{array}{l}\text { El diputado responde y resuelve la cuestión planteada por su inter- } \\
\text { locutor }\end{array}$ \\
\hline \multirow{2}{*}{$\begin{array}{l}\text { COMUNICACIÓN DE } \\
\text { TIPO POLÍTICO-PRO- } \\
\text { PAGANDÍSTICO }\end{array}$} & Respuesta politizada & $\begin{array}{l}\text { El diputado aprovecha la respuesta para defender la posición polí- } \\
\text { tica de su partido en un asunto determinado y/o criticar la de otro } \\
\text { partido rival. }\end{array}$ \\
\hline & Respuesta técnica & $\begin{array}{l}\text { El diputado responde con datos técnicos y no entra en considera- } \\
\text { ciones políticas }\end{array}$ \\
\hline \multirow{2}{*}{ TIEMPO } & Respuesta inmediata & $\begin{array}{l}\text { El diputado contesta dentro de los tres días posteriores al envío } \\
\text { inicial del correo }\end{array}$ \\
\hline & Respuesta demorada & $\begin{array}{l}\text { El diputado contesta a partir del cuarto día tras el envío inicial del } \\
\text { correo }\end{array}$ \\
\hline
\end{tabular}

Fuente: Elaboración propia

La metodología diseñada se completa, en su vertiente cualitativa, con una serie de entrevistas en profundidad a una muestra de nueve diputados integrantes de la delegación española en el Parlamento Europeo en su VII legislatura (2009-2014). La muestra incluye diputados de todos los grupos parlamentarios integrantes de la cámara: Partido Popular Europeo, Alianza Progresista de Socialistas y Demócratas en el Parlamento Europeo, Los Verdes-Alianza Libre Europea, Alianza de los Demócratas y Liberales por Europa, Izquierda Unitaria Europea-Izquierda Verde Nórdica y Grupo de No Inscritos.

El objetivo a la hora de aplicar esta técnica de investigación cualitativa ha sido enriquecer la información sobre el objeto de estudio y servir de complemento y ayuda en la tarea posterior de análisis e interpretación de los resultados obtenidos 
en el trabajo de campo. Las entrevistas se han transcrito y posteriormente se ha aplicado un análisis de contenido sobre las respuestas que ha permitido conocer las motivaciones, preferencias y actitudes expresadas por los diputados en cuanto al objeto de estudio.

\section{Resultados}

Tal como señala el cuadro 2, los diputados de la delegación española en el Parlamento Europeo muestran una disponibilidad absoluta a la hora de publicar una cuenta de email a través de la cual contactar con ellos. Todos los integrantes del universo (54) facilitan una dirección de correo electrónico a través de la ficha personal (Fuente A) alojada en el sitio web de la institución parlamentaria (www.europarl.europa.eu), por lo que el universo coincide con la muestra. Conviene señalar que, en este punto, los eurodiputados españoles cumplen en su totalidad las recomendaciones de la Unión Interparlamentaria Internacional (2009) para que los sitios web parlamentarios incluyan direcciones de email o formularios para que los ciudadanos puedan comunicarse con los representantes públicos. Por ese motivo, no ha sido necesario consultar las fuentes B y C para componer la muestra, ya que todas las direcciones de los integrantes del universo estaban disponibles a través de la fuente $\mathrm{A}$.

Tal como se ha especificado en el punto 2, la muestra se ha revisado y actualizado antes y durante cada una de las oleadas. El desarrollo de esta tarea ha sido especialmente relevante antes de la ejecución de la tercera y última oleada, pues la celebración de las elecciones al Parlamento Europeo del 25 de mayo de 2014 ha modificado la composición y el número de integrantes de cada uno de los grupos parlamentarios. No obstante, la metodología de análisis aplicada ha sido la misma a lo largo de la última oleada del trabajo de campo. Asimismo, los cambios en la muestra han sido mínimos, ya que los grupos parlamentarios no han variado su estructura y el 40 por ciento de sus integrantes (21 de 54 diputados) ha mantenido su acta parlamentaria tras la convocatoria electoral.

El análisis de los datos del cuadro 2 muestra índices de respuesta relativamente bajos en cada una de las tres fases del trabajo de campo. En la primera y segunda oleada, el índice de respuesta ha sido del 20,3 por ciento y del 14,8 por ciento, respectivamente. El mejor escenario posible se ha registrado en la tercera y última oleada, donde han respondido el 55,5 por ciento de los eurodiputados españoles. A pesar de representar el mejor registro de todo el trabajo de campo, los datos revelan que, aún en este escenario, el 44,5 por ciento de los integrantes de la muestra han permanecido en silencio y no han respondido a las cuestiones planteadas por los ciudadanos a través del email.

Según las opiniones expresadas por los diputados europeos durante las entrevistas personales, es posible que detrás de las razones que justifican estos índices de respuesta se encuentre el elevado volumen de correos que reciben a través del email cada día, la falta de tiempo para responder a todos ellos y la intensidad del trabajo parlamentario. 
El análisis de los índices de respuesta también indica que los diputados de las delegación española en el Parlamento Europeo responden más cuando el contenido de los mensajes remitidos por los ciudadanos contiene cuestiones que se leen y resuelven de forma sencilla y que apenas requieren esfuerzo para aportar la información solicitada por el interlocutor. El índice de respuesta de los Mensajes-Función (MF) de la tercera oleada (55,5 por ciento) duplica e incluso triplica los índices de respuesta de los Mensajes Desarrollo (MD) de la primera (20,3 por ciento) y segunda oleada (14,8 por ciento). Los resultados se pueden observar de forma gráfica en el cuadro 3.

Cuadro 2. Disponibilidad para publicar una cuenta de email a través de las fuentes consultadas e índices de respuesta obtenidos a lo largo de las 3 oleadas del trabajo de campo.

\begin{tabular}{|c|c|c|c|c|c|c|c|c|c|}
\hline & \multicolumn{3}{|l|}{$1^{a}$ oleada } & \multicolumn{3}{|l|}{$2^{\mathrm{a}}$ oleada } & \multicolumn{3}{|l|}{$3^{\mathrm{a}}$ oleada } \\
\hline & & \begin{tabular}{|l|} 
Con \\
$@$
\end{tabular} & $\begin{array}{l}\text { Respon- } \\
\text { den }\end{array}$ & & $\begin{array}{l}\text { Con } \\
@\end{array}$ & $\begin{array}{l}\text { Respon- } \\
\text { den }\end{array}$ & & $\begin{array}{l}\text { Con } \\
@\end{array}$ & $\begin{array}{l}\text { Respon- } \\
\text { den }\end{array}$ \\
\hline \multirow{5}{*}{$\begin{array}{l}\text { PARLA- } \\
\text { MENTO } \\
\text { EURO- } \\
\text { PEO }\end{array}$} & $\begin{array}{l}\text { Grupo del Partido } \\
\text { Popular Europeo } \\
\text { (Demócrata - Cris- } \\
\text { tianos) }\end{array}$ & 25 & $5(20 \%)$ & $\begin{array}{l}\text { Grupo del Partido } \\
\text { Popular Europeo } \\
\text { (Demócrata-Cris- } \\
\text { tianos) }\end{array}$ & 25 & $2(8 \%)$ & $\begin{array}{l}\text { Grupo del Partido } \\
\text { Popular Europeo } \\
\text { (Demócrata - Cris- } \\
\text { tianos) }\end{array}$ & 17 & 9 (52’9\%) \\
\hline & $\begin{array}{l}\text { Grupo de la Alianza } \\
\text { Progresista de So- } \\
\text { cialistas y } \\
\text { Demócratas en el } \\
\text { Parlamento Euro- } \\
\text { peo }\end{array}$ & 23 & $6(26 \%)$ & $\begin{array}{l}\text { Grupo de la Alianza } \\
\text { Progresista de So- } \\
\text { cialistas y } \\
\text { Demócratas en el } \\
\text { Parlamento Euro- } \\
\text { peo }\end{array}$ & 23 & 3 (13\%) & $\begin{array}{l}\text { Grupo de la Alianza } \\
\text { Progresista de So- } \\
\text { cialistas y } \\
\text { Demócratas en el } \\
\text { Parlamento Euro- } \\
\text { peo }\end{array}$ & 14 & $7(50 \%)$ \\
\hline & $\begin{array}{l}\text { Grupo de la Alianza } \\
\text { de los Demócratas } \\
\text { y Liberales por Eu- } \\
\text { ropa. }\end{array}$ & 2 & 0 & $\begin{array}{l}\text { Grupo de la Alianza } \\
\text { de los Demócratas } \\
\text { y Liberales por Eu- } \\
\text { ropa. }\end{array}$ & 2 & $1(50 \%)$ & $\begin{array}{l}\text { Grupo de la Alianza } \\
\text { de los Demócratas y } \\
\text { Liberales por Eu- } \\
\text { ropa. }\end{array}$ & 8 & 7 (87’5\%) \\
\hline & $\begin{array}{l}\text { Grupo de los Verdes } \\
\text { / Alianza Libre Eu- } \\
\text { ropea }\end{array}$ & 2 & 0 & $\begin{array}{l}\text { Grupo de los Verdes } \\
\text { / Alianza Libre Eu- } \\
\text { ropea }\end{array}$ & 2 & $1(50 \%)$ & $\begin{array}{l}\text { Grupo de los Verdes } \\
\text { / Alianza Libre Eu- } \\
\text { ropea }\end{array}$ & 4 & $1(25 \%)$ \\
\hline & $\begin{array}{l}\text { Grupo Confederal } \\
\text { de la Izquierda Uni- } \\
\text { taria Europea / } \\
\text { Izquierda Verde } \\
\text { Nórdica }\end{array}$ & 1 & 0 & $\begin{array}{l}\text { Grupo Confederal } \\
\text { de la Izquierda Uni- } \\
\text { taria Europea / } \\
\text { Izquierda Verde } \\
\text { Nórdica }\end{array}$ & 1 & 0 & $\begin{array}{l}\text { Grupo Confederal } \\
\text { de la Izquierda Uni- } \\
\text { taria Europea / } \\
\text { Izquierda Verde } \\
\text { Nórdica }\end{array}$ & 11 & 6 (54’5\%) \\
\hline
\end{tabular}




\begin{tabular}{|c|c|c|c|c|c|c|c|c|c|}
\hline & \multicolumn{3}{|l|}{$1^{\mathrm{a}}$ oleada } & \multicolumn{3}{|l|}{$2^{a}$ oleada } & \multicolumn{3}{|c|}{$3^{\mathrm{a}}$ oleada } \\
\hline & & $\begin{array}{l}\text { Con } \\
@\end{array}$ & $\begin{array}{l}\text { Respon- } \\
\text { den }\end{array}$ & & $\begin{array}{l}\text { Con } \\
@\end{array}$ & $\begin{array}{l}\text { Respon- } \\
\text { den }\end{array}$ & & $\begin{array}{l}\text { Con } \\
@\end{array}$ & $\begin{array}{l}\text { Respon- } \\
\text { den }\end{array}$ \\
\hline \multirow{2}{*}{$\begin{array}{l}\text { PARLA- } \\
\text { MENTO } \\
\text { EURO- } \\
\text { PEO }\end{array}$} & No Inscritos & 1 & 0 & No Inscritos & 1 & $1(100 \%)$ & & & \\
\hline & TOTAL & 54 & $\begin{array}{l}11 \\
\left(20^{\prime} 3 \%\right)\end{array}$ & TOTAL & 54 & \begin{tabular}{|l}
8 \\
$\left(14^{\prime} 8 \%\right)$
\end{tabular} & TOTAL & 54 & \begin{tabular}{|l}
30 \\
$\left(55^{\prime} 5 \%\right)$
\end{tabular} \\
\hline
\end{tabular}

Fuente: Elaboración propia

Cuadro 3. Índice de respuesta de los diputados de la delegación española en el Parlamento Europeo a lo largo de las tres oleadas del trabajo de campo.

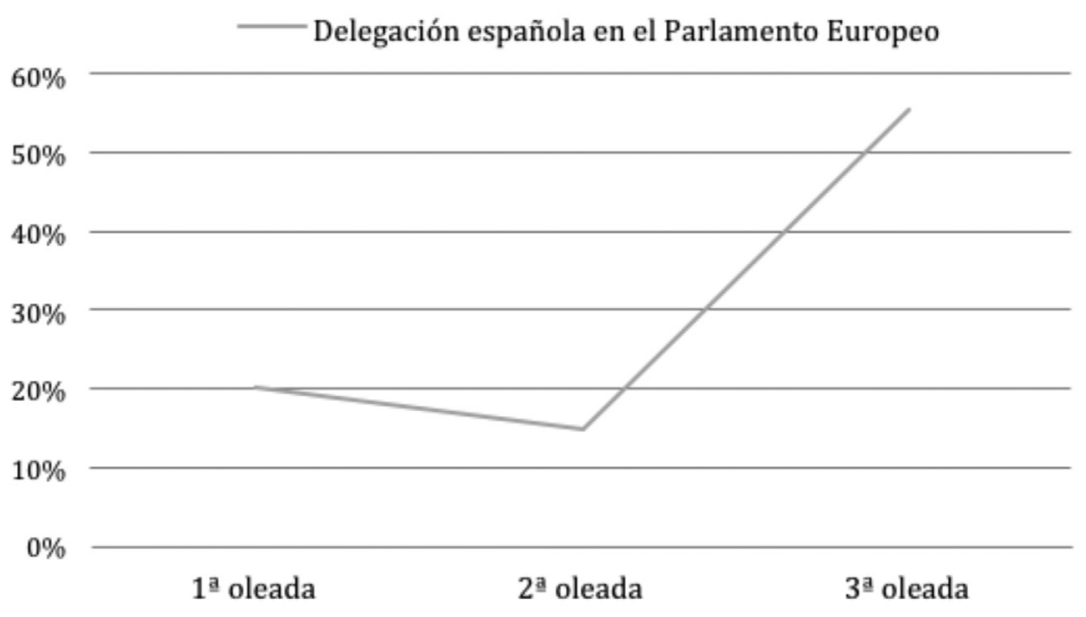

Fuente: Elaboración propia

Del análisis de los datos también se desprende que los grupos minoritarios no demuestran realizar un uso más intenso del email respecto a las formaciones mayoritarias para comunicarse con los ciudadanos. Tal como refleja el cuadro 2, en la primera oleada no se ha registrado ninguna respuesta por parte de los diputados de las formaciones minoritarias. En la segunda oleada, en cambio, todos los grupos minoritarios, a excepción del Grupo Confederal de la Izquierda Unitaria Europea/Izquierda Verde Nórdica superan los registros del Partido Popular Europeo y de la Alianza Progresista de Socialistas y Demócratas. En la tercera y última oleada la tendencia es similar, con todas las formaciones minoritarias, con la salvedad en este caso del Grupo de los Verdes/Alianza Libre Europea, superando los porcentajes obtenidos por parte de los dos 
grupos mayoritarios en la Eurocámara. Así, tal como recoge el cuadro 4, las formaciones minoritarias sí muestran un mejor comportamiento pero su diligencia en el uso del email para relacionarse con el ciudadano no se observa de forma sostenida y consistente en todas ellas.

El análisis de contenido categorial revela un elevado nivel de calidad en las respuestas que se han obtenido por parte de los eurodiputados españoles. A pesar de los bajos índices registrados, aquellos que responden demuestran un nivel de interés e implicación más que aceptable a la hora de responder y resolver el asunto formulado por su interlocutor. Según la categoría contenido, la más relevante para este análisis cualitativo, la media de respuestas argumentadas a lo largo de las tres fases del trabajo de campo ha sido del 95,3 por ciento (90 por ciento en la primera oleada, 100 por ciento en la segunda y 96 por ciento en la tercera, respectivamente).

El análisis de los datos también indica que los eurodiputados españoles recurren a la figura de su asistente personal para responder a la correspondencia electrónica. El porcentaje medio de respuestas personalizadas ha sido del 51,6 por ciento (75 por ciento en la primera oleada, 64 por ciento en la segunda y 16 por ciento en la tercera oleada, respectivamente). Esto significa que en el 48,4 por ciento de los casos, la respuesta ha sido elaborada por una persona del equipo de la oficina del eurodiputado. Sobre este punto, los parlamentarios que participaron en las entrevistas personales reconocieron disponer de al menos un asistente que se encargaba, entre otras tareas, de gestionar el correo electrónico del eurodiputado en total coordinación con él.

Cuadro 4. Índice de respuesta por grupo parlamentario en las tres oleadas del trabajo de campo

\section{Delegación española en el Parlamento Europeo}

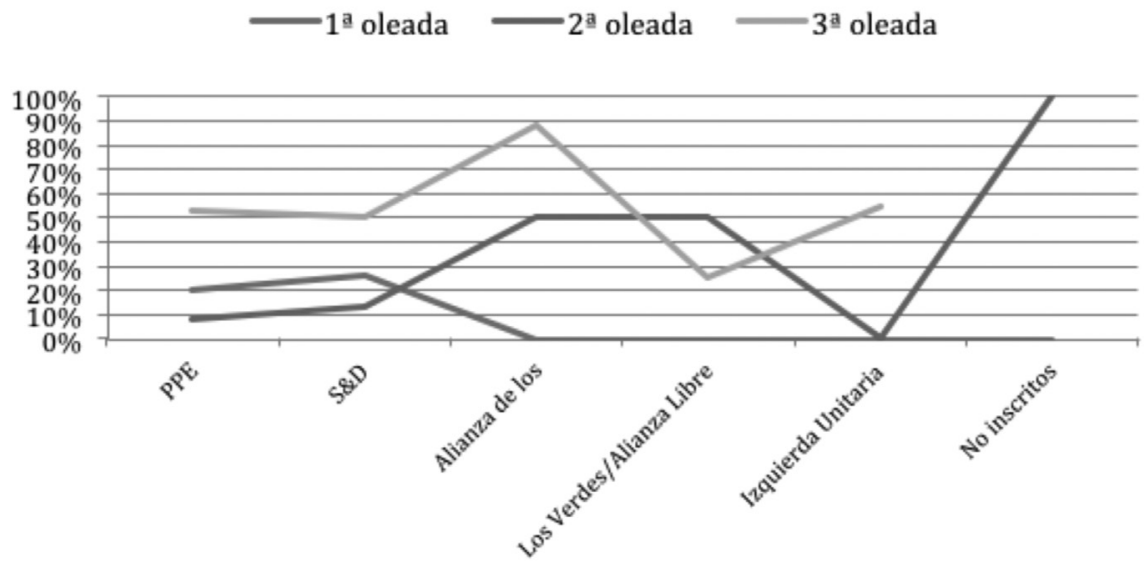

Fuente: Elaboración propia 
Sea por este hecho o no, los europarlamentarios españoles tardan una media de 7,2 días en responder a los ciudadanos que se han dirigido a ellos. Han pasado de los 13 días de media de la primera oleada hasta los 4 de la segunda y los 4,6 de la tercera. Kalman y Rafaeli (2005:7) aseguran que los mensajes que requieren respuesta deben ser contestados tan pronto como sea posible, ya que si se procrastina en esta tarea se corre el riesgo de abandonarla, con la consiguiente percepción negativa para el receptor. Así, se considera que la inmediatez de la respuesta aumenta su nivel de calidad respecto a aquellas otras demoradas por lo que, en el análisis que nos ocupa, la calidad de la respuesta se resiente en la categoría tiempo.

El análisis de contenido categorial también revela que las respuestas de los eurodiputados españoles son mayoritariamente técnicas (73 por ciento y 62,5 por ciento, en la primera y segunda oleada, respectivamente). En aquellos casos en los que emplean los recursos de la comunicación de tipo político-propagandístico (27 por ciento de las respuestas en la primera oleada y 37,5 por ciento en la segunda, respectivamente) los eurodiputados lo hacen para atacar o criticar las políticas de la Unión Europea o del Parlamento Europeo en su conjunto, pero no para censurar las actuaciones del resto de grupos parlamentarios. En la tercera oleada no se ha contemplado esta categoría por el carácter eminentemente funcional de las respuestas obtenidas.

\section{Discusión y conclusiones}

El análisis de los resultados que se ha realizado permite confirmar la primera de las hipótesis, porque el porcentaje de respuesta de los eurodiputados españoles ha sido relativamente bajo en las tres oleadas desarrolladas. El análisis de los datos señala índices de respuesta ligeramente superiores al 50 por ciento de la muestra solo en la tercera y última oleada, ante el envío de los llamados Mensajes-Función. Las dos oleadas anteriores revelan porcentajes que apenas llegan al 20 por ciento, lo que impide apuntar un uso generalizado del email para comunicarse con los ciudadanos que se dirigen a ellos a través de esta vía. Sobre este punto, solo los índices de respuesta de la tercera oleada son superiores a los registros obtenidos por Dader (2003: 312) y Campos (2011: 385) en trabajos previos realizados durante 1999, 2001, 2004 y 2008 en el marco del Congreso de los Diputados y el Senado, y en los que los índices de respuesta de los diputados con cuenta de email pública no superaron, en ninguno de los casos, el 33 por ciento.

El análisis de resultados también permite refutar la segunda de las hipótesis planteadas, ya que los diputados de los grupos parlamentarios minoritarios de la delegación española en el Parlamento Europeo no demuestran realizar un uso más intenso del email respecto a los grupos mayoritarios para relacionarse e interactuar con la ciudadanía. No obstante, y a pesar de no tratarse de un comportamiento uniforme en todas las formaciones, los grupos minoritarios sí muestran un mejor comportamiento en este aspecto, si bien no permite confirmar la hipótesis al no hacerse extensivo a todos los grupos minoritarios de la delegación española en la Eurocámara. 
En el análisis cuantitativo de los índices de respuesta conviene reconocer las limitaciones del email no en cuanto a su capacidad para mantener una interacción muy próxima al diálogo conversacional (Vela Delfa, 2005: 730; Yus Ramos, 2010: 728), sino respecto a la posible saturación de las bandejas de entrada de las cuentas de email oficiales de los diputados a causa de las campañas de bombardeo masivo (Andueza y del Cerro, 2015: 178). Esta posible saturación conllevaría la preferencia de los diputados por otras herramientas de comunicación 2.0 en su relación con los ciudadanos (Álvarez y Rodríguez, 2014: 272).

A pesar de los bajos índices de respuesta, el nivel de calidad de las contestaciones obtenidas es elevado. Los diputados resuelven la cuestión planteada por su interlocutor en la gran mayoría de casos, si bien demoran en exceso la respuesta, pudiendo provocar inquietud o insatisfacción en su interlocutor.

En el escenario analizado, resulta complicado plantearse el empoderamiento de los ciudadanos en la participación de los asuntos públicos si solo una minoría de diputados responden a la interacción ciudadana vía email generando, en consecuencia, niveles tan limitados de "democracia interpelativa", "ciberdemocracia" (Dader y Campos, 2005: 121). La tecnología avanza a un ritmo y permite ciertos mecanismos para los que las estructuras de la institución parlamentaria objeto de estudio en este trabajo no están preparadas.

A la vista de los resultados, debemos señalar que los diputados de la delegación española en el Parlamento Europeo muestran niveles muy limitados de lo que algunos autores han determinado en llamar democracia 2.0, política 2.0 o actitud 2.0 (Fundación Telefónica, 2013: 6; Fagés Ramió, 2008: 22; Orihuela, 2006: 175). Los resultados de este artículo de investigación se suman a la tendencia dominante en la literatura publicada hasta el momento acerca del objeto de la cuestión, según la cual el diálogo no fluye entre políticos y ciudadanos a través de las vía de comunicación de internet. Estos canales, a pesar de estar preparados para lo contrario, siguen funcionando mayoritariamente de forma unidireccional.

En este sentido, el limitado desarrollo de la democracia 2.0 o política 2.0 en la delegación española en el Europarlamento contrasta con el de la administración electrónica o e-administración en España. Es cierto que la e-administración funciona con un nivel de informatización que, aplicado en la misma medida a la democracia 2.0, la desnaturalizaría completamente. No obstante, el crecimiento alcanzado por la administración electrónica en los últimos años sí debe servir de estímulo para determinar que la democracia 2.0 necesita de un impulso igualmente intenso por parte de todos los actores implicados, comenzando por los partidos políticos y sus representantes en las instituciones públicas (Fundación Orange, 2013: 136; Observatorio Nacional de las Telecomunicaciones y de la Sociedad de la Información. ONTSI, 2014: 200).

En cualquier caso, las conclusiones de este artículo deben ser tomadas como una fotografía fija que describe una realidad en un determinado momento en el tiempo. Nada indica que, tomada en otro lapso, la fotografía hubiese arrojado resul- 
tados diferentes. Asimismo, las conclusiones y resultados deben ser interpretados en su justo alcance y dimensión, que viene determinado por los objetivos y las hipótesis planteadas en la introducción. Que los diputados españoles en el Parlamento Europeo no estén utilizando el correo electrónico para comunicarse y mantener una relación directa con los ciudadanos que se dirigen a ellos a través de esta vía no tiene ninguna implicación ulterior que afecte a su responsabilidad parlamentaria.

\section{Referencias bibliográficas}

Álvarez, D. y Rodríguez, R. (2014): “Parlamentarios 2.0: presencia y actividad de diputados y senadores españoles en las redes sociales”, en Rubio, R. (coord.): Parlamentos Abiertos. Tecnología y redes para la democracia. Madrid: Congreso de los Diputados, pp. 235-276.

Andueza, B. y Del Cerro, R.A (2015): “¿Cómo valoran los diputados su presencia en las redes sociales?: Análisis de la utilización de Internet en el Congreso de los Diputados durante la X Legislatura”, Doxa Comunicación, n. 21, pp. 161-183. Disponible en: http://goo.gl/ijiVLh [Consultado el: 20/12/15].

Arroyo, L. (2012): 10 razones por las que Twitter no sirve para (casi) nada en política. Disponible en: http://goo.gl/ZVlpqM [Consultado el: 22/05/14].

Asociación para la Investigación de los Medios de Comunicación. AIMC (2015): Audiencia de Internet. Abril/mayo 2015. EGM. Disponible en: http://goo.gl/ZugkV [Consultado el: 5/08/15].

Bardin, L. (1986): Análisis de contenido. Madrid: Akal Universitaria.

Campos, E. (2011): El desarrollo de la ciberdemocracia en el Congreso de los Diputados: La comunicación e interacción entre ciudadanos y parlamentarios a través de internet (2004-2008). Tesis doctoral. Universidad Complutense, Madrid. Disponible en: http://goo.gl/YFBnjA [Consultado el: 8/03/13].

Castells, M. (2003): La era de la información. El poder de la identidad. Madrid: Alianza Editorial.

Cebrián E.; Vázquez, T. y Olabarrieta, A. (2013): “¿Participación y democracia en los medios sociales?: El caso de Twitter en las elecciones vascas de 2012", adComunica. Revista Cientifica de Estrategias, Tendencias e Innovación en Comunicación, n. 6, pp. 39-63. Disponible en: http://goo.gl/0Zz9wy [Consultado el: 09/0514].

Congosto, M. L. (2011): “Del 15-M a la acampada de Sol”, BarriBlog. Disponible en: http://goo.gl/dHNHYW [Consultado el: 0407/14].

Dader, J. L. (2003): “Ciberdemocracia y comunicación política virtual: el futuro de la ciudadanía electrónica tras la era de la televisión”, en Berrocal, S. (coord.): Comunicación política en televisión y nuevos medios. Barcelona: Ariel Comunicación, pp. 309-342.

74 | n 22, pp. 57-78 | doxa.comunicación 
Dader, J. L. y Campos, E. (2005): “Internet parlamentario en España (1999-2005): los recursos para el contacto y su uso, con una comparación europea”, ZER, n. 20, pp. 105-132. Disponible en: http://goo.gl/q8Vwqj [Consultado el: 13/01/10].

Dans, E. (2011): “Entendiendo la \#spanishrevolution”, El Blog de Enrique Dans. Disponible en: http://goo.gl/SjBvz [Consultado el: 0406/14].

Dapena, B. y Rúas Araújo, J. (2011): “Los diputados del Parlamento Gallego en Facebook”, Redmarka. Revista Académica de Marketing Aplicado y Comunicación, n. 7, pp. 77-106. Disponible en: http://goo.gl/W0exqC [Consultado el: 20/05/14].

De Vega García, P. (1996): “La crisis de la democracia representativa”, en Tezanos, J. F. (ed.): La democracia post-liberal. Madrid: Editorial Sistema, pp. 75-110.

Fagés-Ramió, R. (2008): "Actitud 2.0: la política más allá de los blogs", en IV Congreso Internet, Derecho y Politica (IDP). Software social y Web 2.0: Implicaciones jurídico-politicas (monográfico en línea). IDP. Revista de Internet, Derecho y Política, n. 7. UOC. Disponible en: http://goo.gl/0UJGK [Consultado el: 05/0514].

Fallows, J. (1996): Breaking the news: how the media undermine American democracy. New York: Pantheon.

Ferreras, E. M. (2011): “Redes sociales y cambio social. El movimiento 15-M y su evolución en Twitter”, Telos. Cuadernos de comunicación e innovación, n. 89, pp. 1-13. Disponible en: http://goo.gl/ChAjGX

Fundación Orange (2013): e-España. Informe anual 2013 sobre el desarrollo de la sociedad de la información en España, pp. 1-234. Disponible en: http://goo.gl/KElrz [Consultado el: 12/06/14].

Fundación Telefónica (2013): Las TIC en el gobierno abierto: transparencia, participación y colaboración. Barcelona: Ariel, pp. 1-120. Disponible en: http://www.fundacion.telefonica.com/es/arte_cultura/publicaciones/detalle/211 [Consultado el: 13/0614].

Gibson R. y Ward S. (1999): "Party democracy on-line: UK parties and new ICTs”, Information Communication and Society, n. 2 (3), pp. 340-367.

Gingras, A. M. (1998): “El impacto de las comunicaciones en las prácticas políticas”, en Gauthier, G.; Gosselin, A. y Mouchon, J. (comps.): Comunicación y política. Barcelona: Gedisa, pp. 31-43.

Gómez Fernández, P. (1999): “El marketing político como producto de la democracia mediática”, en Muñoz Alonso, A. y Rospir, J. I. (eds.): Democracia mediática y campañas electorales. Barcelona: Ariel Comunicación, pp. 89-109.

Grossman, L. (1995): The electronic republic: Reshaping American democracy in the information age. New York: Penguin Books.

Gutiérrez-Rubí, A. (2011): \#15-M: política sin partidos. Artículos y reflexiones. Disponible en: http://goo.gl/krqNFY [Consultado el: 04/0614].

Hart, R. P. (1994): “Easy citizenship: Television's curious legacy”, Annals of the American Academy of Political Social Science, n. 546 (1), pp. 109-120. Disponible en: http://goo.gl/trvoHG [Consultado el: 22/05/14]. 
Higueras, G. (2011): “La justicia amenaza a Mubarak y su fabulosa fortuna”, El País. Disponible en: http://goo.gl/Ewr81r [Consultado el: 13/02/11].

Juris, J. S. (2006): “Movimientos sociales en red. Movimientos globales por una justicia global”, en Castells, M. (ed.): La sociedad red. Una visión global. Madrid: Alianza, pp. 415-439.

Kalman, Y. M. y Rafaeli, S. (2005): “Email chronemics: Unobtrosive profiling of response times”, Proceedings of te 38th Hawaii International Conference on System Sciencies. Disponible en: http://goo.gl/NXBfOs [Consultado el: 20//01/13].

Krippendorff, K. (1990): Metodología de análisis de contenido. Teoría y práctica. Barcelona: Paidós Comunicación.

Lilleker, D. G. y Jackson, N. A. (2008): “Politicians and Web 2.0: the current bandwagon or changing the mindset?", Paper presented at the Politics: Web 2.0 International Conference. April 17-18, 2008. London: Royal Holloway, University of London. Disponible en: http://goo.gl/vfUAqd [Consultado el: 20/04/13].

Lilleker, D. G. y Jackson, N. A. (2009): “Interacting and Representing: can Web 2.0 enhance the roles of an MP?”, ECPR workshop "Parliaments, Parties and Politicians in Cyberspace". Lisbon, April 2009, pp. 1-26.

Maravall, J. M. (2003): El control de los políticos. Madrid: Taurus.

Martinez, G. (2011): "Redes sociales y mundo árabe. Lenguaje y significado", Telos. Cuadernos de comunicación e innovación. Octubre-Diciembre 2011, pp. 1-3. Disponible en: http://goo.gl/qNFpk5 [Consultado el: 20/0514].

Morales, L. (2005): “¿Existe una crisis participativa? La evolución de la participación política y el asociacionismo en España”, Revista Española de Ciencia Política, n. 13, pp. 51-87.

Morozov, E. (2011): El desengaño de internet: los mitos de la libertad en la red. Barcelona: Destino.

Muñoz Alonso, A. (1999): “La democracia mediática”, en Muñoz Alonso, A. y Rospir, J. I. (eds.): Democracia mediática y campañas electorales. Barcelona: Ariel Comunicación, pp. 13-53.

Negroponte, N. (1995): El mundo digital. Barcelona: Ediciones B.

Neveu, E. (1998): “Los programas políticos de la televisión. La década de los 80 o los atolladeros del espectáculo político”, en Gauthier, G.; Gosselin, A. y Mouchon, J. (comps.): Comunicación y política. Barcelona: Gedisa, pp. 153-174.

Newton, K. y Norris, P. (2000): “Confidence in public institutions: faith, culture or performance?”, en Pharr, S. J. y Putnam, R. (eds.): Disaffected democracies. What's troubling with trilateral countries. Princeton: Princeton University Press, pp. 5273.

Norris, P. (2003): “Preaching to the converted? Pluralism, participation and party websites", Party Politics, n. 9 (1), pp. 2145.

Observatorio Nacional de las Telecomunicaciones y de la Sociedad de la Información, ONTSI (2014): La sociedad en red. Informe anual 2013 (edición 2014), pp. 1-281. Disponible en: http://goo.gl/6Umt0v [Consultado el: 08/0914]. 
O'Reilly, T. (2005): What is Web 2.0: Design patterns and business models for the next generation of software. Disponible en: http://goo.gl/m4Xnqm [Consultado el: 14/0212].

Orihuela, J. L. (2006): La revolución de los blogs. Madrid: La esfera de los libros.

- (2011): “España ya tiene su revolución”, eCuaderno. Disponible en: http://goo.gl/pCbfs [Consultado el: 04/06/14].

Papacharisi, Z. (2009): “The citizen is the message. Alternative modes of civic engagement”, en Papacharisi, Z. (comp.): Journalism and citizenship: new agendas in communication. Londres: Routledge, pp. 29-40.

Pérez-Díaz, V. (2003): "De la guerra civil a la sociedad civil: el capital social en España entre los años 30 y los años 90 del S.XX”, en Putnam, R. (ed.): El declive del capital social. Un estudio internacional sobre las sociedades y en sentido comunitario. Barcelona: Galaxia Gutemberg, pp. 427-491.

Pindado, F. (1999): “Evolución de las formas de participación”, en Pindado, F. (ed.): La participación ciudadana en la vida de las ciudades. Barcelona: Ediciones del Serbal, pp. 35-40.

Postman, N. (2001): Divertirse hasta morir. El discurso público en la era del show business. Barcelona: Ediciones de la Tempestad.

Putnam, R. (1995): “Bowling alone: America’s declining social capital”, Journal of democracy, n. 6 (1), pp. 65-78. Disponible en: http://goo.gl/iY7gBW [Consultado el: 28/10/13].

- (2003): “Conclusiones”, en Putnam, R. (ed.): El declive del capital social. Un estudio internacional sobre las sociedades y en sentido comunitario. Barcelona: Galaxia Gutemberg, pp. 623-656.

Reig, D. (2011): “\#yeswecamp: nosotros sí podemos”, El Caparazón. Disponible en: http://goo.gl/r0glF [Consultado el: 04/06/14].

Rheingold, H. (1993): The Virtual Community: Homesteading on the Electronic Frontier. Reading: MA. Addison-Wesley.

Rodríguez Vives, L.; Congosto, M. L. y Giura, A. (2012): Análisis de las elecciones andaluzas del 25M en Twitter. Disponible en: http://goo.gl/kwiP8y [Consultado el: 23/06/12].

Rospir, J. I. (1999): “La globalización de las campañas electorales”, en Muñoz Alonso, A. y Rospir, J. I. (eds.): Democracia mediática y campañas electorales. Barcelona: Ariel Comunicación, pp. 56-88.

Sampedro, V. y Sánchez Duarte, J. M. (2011): La Red era la plaza. Disponible en: http://goo.gl/9xWWIW [Consultado el: 03/06/13].

Sanmartí, J. M. (2003): “Los nuevos agentes políticos en la comunicación televisiva”, Berrocal, S. (coord.): Comunicación política y nuevos medios. Barcelona: Ariel Comunicación, pp. 81-109.

Sartori, G. (1998): Homo videns. La sociedad teledirigida. Madrid: Taurus. 
Scolari, C. (2009): “Después del temblor: la movilización de masas en la época post-SMS”, Hipermediaciones. Disponible en: http://goo.gl/9bq6HF [Consultado el: 04/06/13].

Sinova, J. y Tusell, J. (1997): La crisis de la democracia en España. Ideas para reinventar nuestro sistema político. Madrid: Espasa.

Small, T. (2010): “Canadian Politics in 140 characters: Party Politics in the Twitterverse”, Canadian Parliamentary Review, Autumn 2010, pp. 39-45. Disponible en: http://goo.gl/W7dZzf [Consultado el: 23/05/14].

Torcal, M. (1992): “Análisis dimensional y estudio de valores: el cambio cultural en España”, Revista Española de Investigaciones Sociológicas, n. 58, pp. 97-122.

Túñez, M. y Sixto, J. (2011): “Redes sociales, política y Compromiso 2.0: La comunicación de los diputados españoles en Facebook", Revista Latina de Comunicación Social, n. 66, pp. 1- 25. Disponible en: http://goo.gl/96S8Ct [Consultado el: 30/04/14].

Unión Interparlamentaria Internacional (2009): Directrices para sitios web parlamentarios, pp. 7-32. Disponible en: http://ipu.org/PDF/publications/web-s.pdf [Consultado el: 10/06/10].

Vela Delfa, C. (2005): El correo electrónico: El nacimiento de un nuevo género. Tesis Doctoral. Universidad Complutense de Madrid. Disponible en: http://goo.gl/ceFk4g [Consultado el: 04/05/14].

Yus Ramos, F. (2010): Ciberpragmática 2.0. El uso del lenguaje en internet. Barcelona: Ariel.

Zurutuza Muñoz, C. (2012): “¿De qué habla Rubalcaba cuando tweetea? La campaña del candidato socialista en Twitter para las elecciones del 20N", I Congreso Internacional en Comunicación Política y Estrategias de Campaña. Disponible en: http://goo.gl/e2oWdI [Consultado el: 22/05/14]. 